\title{
Analysis of requirements for modern finishing materials in interior solutions for inpatient facility ward units
}

\author{
Alevtina Balakina ${ }^{1, *}$ and Yury Lempl ${ }^{1}$ \\ ${ }^{1}$ Moscow State University of Civil Engineering (MGSU), 26, Yaroslavskoye Shosse, 129337, \\ Moscow, Russia
}

\begin{abstract}
This article is devoted to the detailed analysis of modern requirements for finishing materials, used in the interiors of inpatient facility ward units. The authors have studied a number of regulatory normative documents acting in the territory of the Russian Federation and analyzed the practical experience of interior decoration of ward units at the health care capital construction facilities. The authors highlight and describe the main requirements for the finishing materials for the inpatient facility ward units and point out the peculiarities of solutions in the interiors. A classification of modern finishing materials (with an indication of their technical characteristics) used in the decoration of interiors of longterm care units is made on the basis of the practical experience studied by the authors taking into account the functional purpose of the premises as well as the variants of visual design of interiors of inpatient facility ward units.
\end{abstract}

\section{Introduction}

With the development and modernization of the healthcare system in the Russian Federation, the issue of ensuring the quality and safety of medical activities carried out in health care institutions is most acute [1,2]. This is particularly true in the case of long-term care facilities, i.e. inpatient facilities.

This paper reviews the requirements for finishing materials used in the design of the interiors of inpatient facility ward units $[3,4,6,7]$.

A number of papers and manuals $[9,10,11,12]$ outline the general requirements for the interior decoration of health care institutions, as well as individual requirements for ward units. A special place in the interior design of health care premises is occupied by units for permanent or temporary stay of patients. In this regard, the requirements for the interiors of wards and public areas of inpatient facilities have a number of peculiarities:

- technology requirements, which in this category of premises are primarily related to furniture and engineering arrangement [11]: functionally equipped medical bedside consoles, functional beds, functional bedside tables and bedside chests, sanitary units, equipped taking into account the requirements of accessibility of the room for people with

\footnotetext{
* Corresponding author: balakinaae@mail.ru
} 
low mobility (the requirements are divided by the criteria of accessibility, informativeness, safety, comfort) [5], special lighting of wards [9,10].

- technical requirements - engineering solutions aimed at ensuring effective and comfortable treatment, including ensuring compliance with regulatory sanitary [3] and fire requirements[8], safety requirements [2], climate control in both wards and public areas, equipment with modern doctor or nurse call systems, equipment with patient monitoring information systems, equipment of wards and other special units with medical gases and others.

- Aesthetic and psychological requirements for this category of facilities play a very important role in creating a comfortable stay and effective treatment. Modern interiors should create a feeling of home comfort and well-being for people with different types of diseases, the possibility, even while being treated in a health care institution to continue their communication with the outside world, as well as, if necessary, to continue to work on their favorite things. Preference is given to options that exclude "wet" processes in the finishing of such rooms.

\section{Materials and methods}

In this paper, the authors have analyzed a number of regulatory documents (including recommendations for interior design of hospitals), adopted in the Russian Federation, to identify typical requirements for finishing materials used in the ward units of health care institutions.

Based on these requirements, the authors studied the practical experience of implementing these requirements at capital construction health care facilities (major repairs, new construction, reconstruction), in particular with the execution of works in the ward units.

\section{Results}

Having synthesized the obtained theoretical and practical experience of interiors decoration of inpatient facility ward units, the authors have prepared Table 1, in which finishing materials (taking into account their compliance with standards, modern technologies, recommendations on coloristic solutions) used in the finishing of inpatient facility ward units, depending on their functional purpose were selected. All offered finishing materials are classified according to the type of finishing works.

Table 1. Finishing materials recommended in the creation of interiors of health care institution ward units.

\begin{tabular}{|c|c|c|c|c|}
\hline $\begin{array}{c}\text { Description } \\
\text { of } \\
\text { the premises }\end{array}$ & Floor finish & Wall decoration & Ceiling finish & Lighting \\
\hline Foyer & $\begin{array}{l}-50401 \quad \text { Forbo } \\
\text { Sphera Evolution } \\
\text { silver white pearl } \\
\text { linoleum }\end{array}$ & $\begin{array}{l}\text { HPL decorative wall } \\
\text { panels, RAL 9010; } \\
\text { HPL decorative wall } \\
\text { panels with wood } \\
\text { texture; } \\
\text { HPL decorative wall } \\
\text { panels with specialty } \\
\text { color: Dulux S 1510- } \\
\text { R80B }\end{array}$ & $\begin{array}{l}\text { - Armstrong } \\
\text { type } \\
\text { suspended } \\
\text { ceiling panels } \\
\text { with } \\
\text { concealed } \\
\text { fixing. } \\
\text { Dimensions: } \\
600 \times 1200 \mathrm{~mm}\end{array}$ & $\begin{array}{l}\text { Luminaires: } \\
-\quad 250 \mathrm{~mm} \\
\text { recessed } \\
\text { ceiling } \\
\text { spotlight; } \\
\text { - Warton LED } \\
\text { ceiling lamp, } \\
\text { dimensions: } \\
\text { 1200x200x50 } \\
\mathrm{mm}\end{array}$ \\
\hline Lobby & - FORBO Sphera & HPL decorative wall & - $\quad$ Armstrong & Luminaires: \\
\hline
\end{tabular}




\begin{tabular}{|c|c|c|c|c|}
\hline & $\begin{array}{l}\text { SD } 550017 \text { ivory } \\
\text { light grey } \\
\text { marmoleum }\end{array}$ & $\begin{array}{l}\text { panels, RAL } 9010 \text {, } \\
\text { HPL decorative wall } \\
\text { panels with wood } \\
\text { texture }\end{array}$ & $\begin{array}{l}\text { type } \\
\text { suspended } \\
\text { ceiling panels } \\
\text { with } \\
\text { concealed } \\
\text { fixing. } \\
\text { Dimensions: } \\
600 \times 1200 \mathrm{~mm}\end{array}$ & $\begin{array}{l}\text { - 250mm } \\
\text { recessed } \\
\text { ceiling } \\
\text { - Warton LED } \\
\text { ceiling lamp, } \\
\text { dimensions: } \\
\text { 1200x200x50 } \\
\text { mm } \\
\text {-Kink Light } \\
\text { Tor 08223.19P } \\
\text { Pendant LED } \\
\text { luminaire }\end{array}$ \\
\hline $\begin{array}{l}\text { Waiting } \\
\text { area }\end{array}$ & $\begin{array}{l}-550037 \text { Forbo } \\
\text { Sphera SD } \\
\text { homogeneous } \\
\text { China blue } \\
\text { linoleum } \\
-550017 \text { Forbo } \\
\text { Sphera SD ivory } \\
\text { linoleum }\end{array}$ & $\begin{array}{l}\text { HPL decorative wall } \\
\text { panels with wood } \\
\text { texture } \\
\text {-Wall painting with } \\
\text { specialty color: Du- } \\
\text { lux S 1510-R80B }\end{array}$ & $\begin{array}{l}\text {-Ceiling made } \\
\text { of gypsum } \\
\text { plasterboard } \\
\text { painted matt } \\
\text { RAL } 9016 \\
\text { acrylic paint }\end{array}$ & $\begin{array}{l}\text { Luminaires: } \\
\text { Warton LED } \\
\text { ceiling lamp, } \\
\text { dimensions: } \\
\text { 1200x200x50 } \\
\text { mm } \\
\text {-Decorative } \\
\text { LED luminaire } \\
\text { with frosted } \\
\text { diffuser, } \\
\mathrm{D}=1400 \mathrm{~mm}\end{array}$ \\
\hline $\begin{array}{l}\text { Day } \\
\text { care } \\
\text { area }\end{array}$ & $\begin{array}{lr}-550037 & \text { Forbo } \\
\text { Sphera SD } \\
\text { homogeneous } \\
\text { China blue } \\
\text { linoleum } \\
-550017 \text { Forbo } \\
\text { Sphera Sd ivory } \\
\text { linoleum }\end{array}$ & $\begin{array}{l}\text { HPL decorative wall } \\
\text { panels with wood } \\
\text { texture } \quad \text {-Wall } \\
\text { painting, color RAL } \\
9016, \\
\text {-Wall painting with } \\
\begin{array}{l}\text { Dulux S 1510-R80B } \\
\text { color }\end{array}\end{array}$ & $\begin{array}{l}\text { - Armstrong } \\
\text { type } \\
\text { suspended } \\
\text { ceiling panels } \\
\text { with } \\
\text { concealed } \\
\text { fixing. } \\
\text { Dimensions: } \\
600 \times 1200 \mathrm{~mm}\end{array}$ & $\begin{array}{l}\text { Luminaires: } \\
\text {-Warton LED } \\
\text { ceiling lamp, } \\
\text { dimensions: } \\
\text { 1200x600x50 } \\
\mathrm{mm}\end{array}$ \\
\hline Corridor & $\begin{array}{l}-50405 \text { Forbo } \\
\text { Sphera Evolution } \\
\text { bronze pearl } \\
\text { marmoleum }\end{array}$ & \begin{tabular}{lr} 
Wall painting & with \\
specialty & color: \\
Duluxe & $90 \mathrm{BG}$ \\
$56 / 125$, & Wall \\
painting & with \\
specialty & color: \\
Dulux S 1510-R80B, & -Wall painting, color \\
\multicolumn{2}{l}{ RAL 9010 } \\
\end{tabular} & $\begin{array}{l}\text { - Armstrong } \\
\text { type } \\
\text { suspended } \\
\text { ceiling panels } \\
\text { with } \\
\text { concealed } \\
\text { fixing. } \\
\text { Dimensions: } \\
600 \times 1200 \mathrm{~mm} \\
\end{array}$ & $\begin{array}{l}\text { Luminaires: } \\
\text {-Warton LED } \\
\text { ceiling lamp, } \\
\text { dimensions: } \\
\text { 1200x200x50 } \\
\mathrm{mm}\end{array}$ \\
\hline Lift lobby & 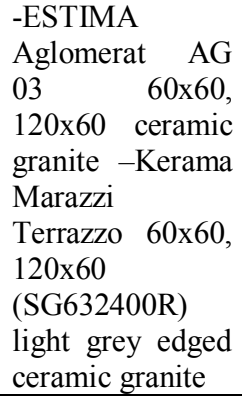 & $\begin{array}{l}\text { HPL decorative wall } \\
\text { panels, specialized } \\
\text { color: Dulux } 90 \mathrm{BG} \\
56 / 125, \quad-\quad \text { HPL } \\
\text { decorative } \\
\text { panels, color } \\
9010\end{array}$ & $\begin{array}{l}\text { - Armstrong } \\
\text { type } \\
\text { suspended } \\
\text { ceiling panels } \\
\text { with } \\
\text { concealed } \\
\text { fixing. } \\
\text { Dimensions: } \\
600 \times 1200 \mathrm{~mm}\end{array}$ & $\begin{array}{l}\text { Luminaires: } \\
\text {-Warton LED } \\
\text { ceiling lamp, } \\
\text { dimensions: } \\
\text { 1200x600x50 } \\
\mathrm{mm}\end{array}$ \\
\hline $\begin{array}{l}\text { Ward per } 1 \\
\text { bed }\end{array}$ & $\begin{array}{l}-50432 \text { Forbo } \\
\text { Sphera Evolution } \\
\text { homogeneous } \\
\text { silver white } \\
\text { linoleum }\end{array}$ & $\begin{array}{l}\text { HPL decorative wall } \\
\text { panels with wood } \\
\text { texture } \\
\text { HPL decorative wall } \\
\text { panels with specialty }\end{array}$ & $\begin{array}{l}\text { Armstrong } \\
\text { type } \\
\text { suspended } \\
\text { ceiling panels } \\
\text { with } \\
\end{array}$ & $\begin{array}{l}\text { Luminaires: -- } \\
\text { Recessed } \\
\text { LED } \\
\text { luminaire } \\
1200 \times 600 \times 100\end{array}$ \\
\hline
\end{tabular}




\begin{tabular}{|c|c|c|c|c|}
\hline & & $\begin{array}{l}\text { color: Duluxe } 50 \mathrm{GY} \\
66 / 111 \\
\text {-wall painting, RAL } \\
9010\end{array}$ & $\begin{array}{l}\text { concealed } \\
\text { fixing. } \\
\text { Dimensions: } \\
600 \times 1200 \mathrm{~mm}\end{array}$ & \\
\hline $\begin{array}{l}\text { Ward per } 2 \\
\text { beds }\end{array}$ & $\begin{array}{lr}-550008 & \text { Forbo } \\
\text { Sphera } & \text { SD } \\
\text { homogeneous } \\
\text { silver } & \text { grey } \\
\text { linoleum } & \end{array}$ & $\begin{array}{l}\text {-HPL decorative wall } \\
\text { panels, RAL 9010, } \\
\text {-HPL decorative wall } \\
\text { panels with wood } \\
\text { texture } \\
\text {-wall painting with } \\
\text { specialty color: } \\
\text { Dulux 50GY 66/111 }\end{array}$ & $\begin{array}{l}\text { - Armstrong } \\
\text { type } \\
\text { suspended } \\
\text { ceiling panels } \\
\text { with } \\
\text { concealed } \\
\text { fixing. } \\
\text { Dimensions: } \\
600 \times 1200 \mathrm{~mm}\end{array}$ & $\begin{array}{l}\text { Luminaires: - } \\
\text { Recessed } \\
\text { LED } \\
\text { luminaire } \\
\text { 1200x600x100 }\end{array}$ \\
\hline Sanitary Unit & $\begin{array}{l}\text {-Kerama Marazzi } \\
1544 \\
\text { Kaleidoscope } \\
\text { white tile }\end{array}$ & $\begin{array}{ll}\text {-Kerama } & \text { Marazzi } \\
\text { tile, } & \\
11120 \mathrm{R} & \text { Marceau } \\
\text { white } & \text { edged, } \\
595 \times 295 \mathrm{~mm} & \end{array}$ & $\begin{array}{l}-\quad \text { Armstrong } \\
\text { type } \\
\text { suspended } \\
\text { ceiling panels } \\
\text { with } \\
\text { concealed } \\
\text { fixing. } \\
\text { Dimensions: } \\
600 \times 1200 \mathrm{~mm} \\
\end{array}$ & $\begin{array}{l}\text { Luminaires: } \\
-\quad 250 \mathrm{~mm} \\
\text { recessed } \\
\text { ceiling }\end{array}$ \\
\hline $\begin{array}{l}\text { Sanitary Unit } \\
\text { for people } \\
\text { with limited } \\
\text { mobility }\end{array}$ & $\begin{array}{l}\text { - ESTIMA AG01 } \\
\text { light tiles, 60x60 }\end{array}$ & $\begin{array}{l}\text {-Kerama Marazzi } \\
\text { tile, 90-11, Accord } \\
\text { Beige. light facet, } \\
8.5 \times 28.5-\text { Kerama } \\
\text { Marazzi tiles, 90-12, } \\
\text { Accord green facet, } \\
8.5 \times 28.5\end{array}$ & $\begin{array}{l}\quad \text { Armstrong } \\
\text { type } \\
\text { suspended } \\
\text { ceiling panels } \\
\text { with } \\
\text { concealed } \\
\text { fixing. } \\
\text { Dimensions: } \\
600 \times 1200\end{array}$ & $\begin{array}{l}\text { Luminaires: } \\
-\quad 150 \mathrm{~mm} \\
\text { recessed } \\
\text { ceiling } \\
\text { spotlight }\end{array}$ \\
\hline $\begin{array}{l}\text { Main } \\
\text { inpatient } \\
\text { facility } \\
\text { head's } \\
\text { office }\end{array}$ & $\begin{array}{lr}-550008 & \text { Forbo } \\
\text { Sphera } & \text { SD } \\
\text { homogeneous } \\
\text { silver } \\
\text { linoleum }\end{array}$ & $\begin{array}{l}\text {-HPL decorative wall } \\
\text { panels with wood } \\
\text { texture } \\
\text { HPL decorative wall } \\
\text { panels with specialty } \\
\text { color: NCS S 1020- } \\
\text { B10G } \\
\text {-Wall painting, RAL } \\
9010\end{array}$ & $\begin{array}{l}\quad \text { Armstrong } \\
\text { type } \\
\text { suspended } \\
\text { ceiling panels } \\
\text { with } \\
\text { concealed } \\
\text { fixing. } \\
\text { Dimensions: } \\
600 \times 1200 \mathrm{~mm} \\
\end{array}$ & $\begin{array}{l}\text { Luminaires: } \\
-250 \quad \mathrm{~mm} \\
\text { recessed } \\
\text { ceiling } \\
\text { spotlight, } \\
-1200 \times 600 \\
\text { recessed } \\
\text { LED } \\
\text { luminaire } \\
\end{array}$ \\
\hline $\begin{array}{l}\text { Examination } \\
\text { unit }\end{array}$ & $\begin{array}{l}\text { - } 550005 \text { Forbo } \\
\text { Sphera SD } \\
\text { homogeneous } \\
\text { dark neutral grey } \\
\text { linoleum }\end{array}$ & $\begin{array}{l}\text {-HPL decorative wall } \\
\text { panels with wood } \\
\text { texture } \\
\text { HPL decorative wall } \\
\text { panels with specialty } \\
\text { color: DULUX } \\
52 Y Y \text { 89/117 }\end{array}$ & $\begin{array}{l}-\quad \text { Armstrong } \\
\text { type } \\
\text { suspended } \\
\text { ceiling panels } \\
\text { with } \\
\text { concealed } \\
\text { fixing. } \\
\text { Dimensions: } \\
600 \times 1200 \\
\end{array}$ & $\begin{array}{l}\text { Luminaires: -- } \\
\text { Recessed LED } \\
\text { luminaire } \\
\text { 1200x600x100 } \\
-\quad 250 \mathrm{~mm} \\
\text { recessed } \\
\text { ceiling }\end{array}$ \\
\hline
\end{tabular}

Based on the results of the study presented in Table 1, the authors have prepared options for the interior design of the inpatient facility ward units, depending on their functional purpose, presented in Figures 1-13. The proposed interior solutions fully take into account all the requirements put forward to the design of the inpatient facility ward units [1-13]. 


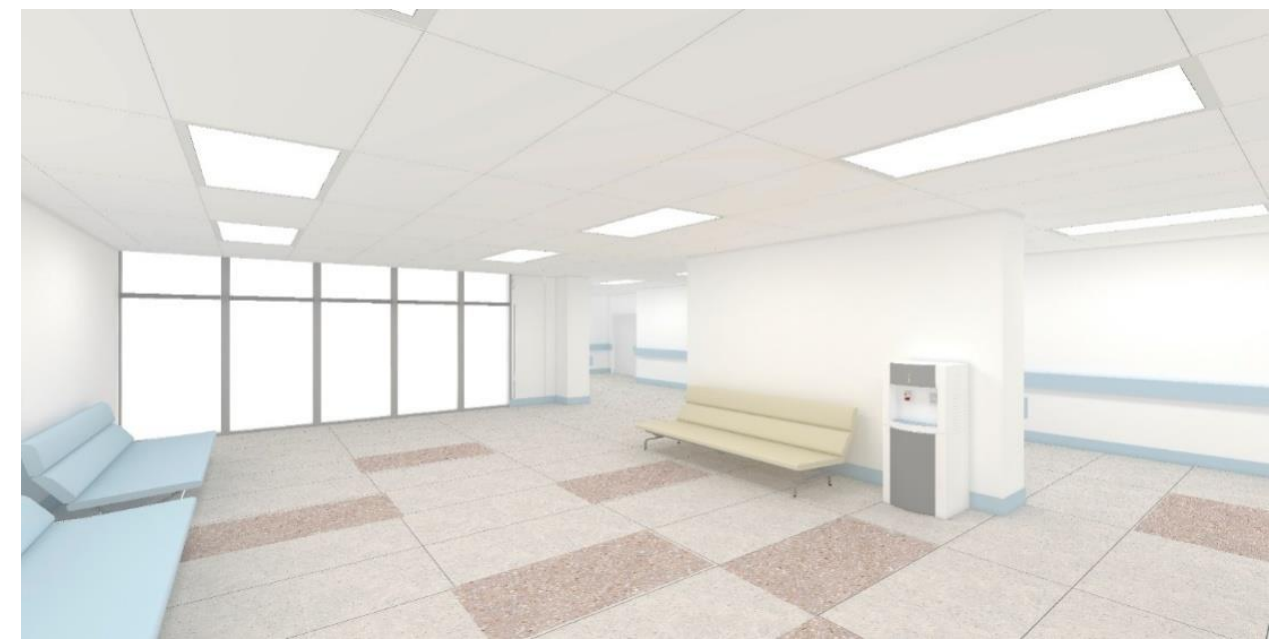

Fig. 1. Foyer.

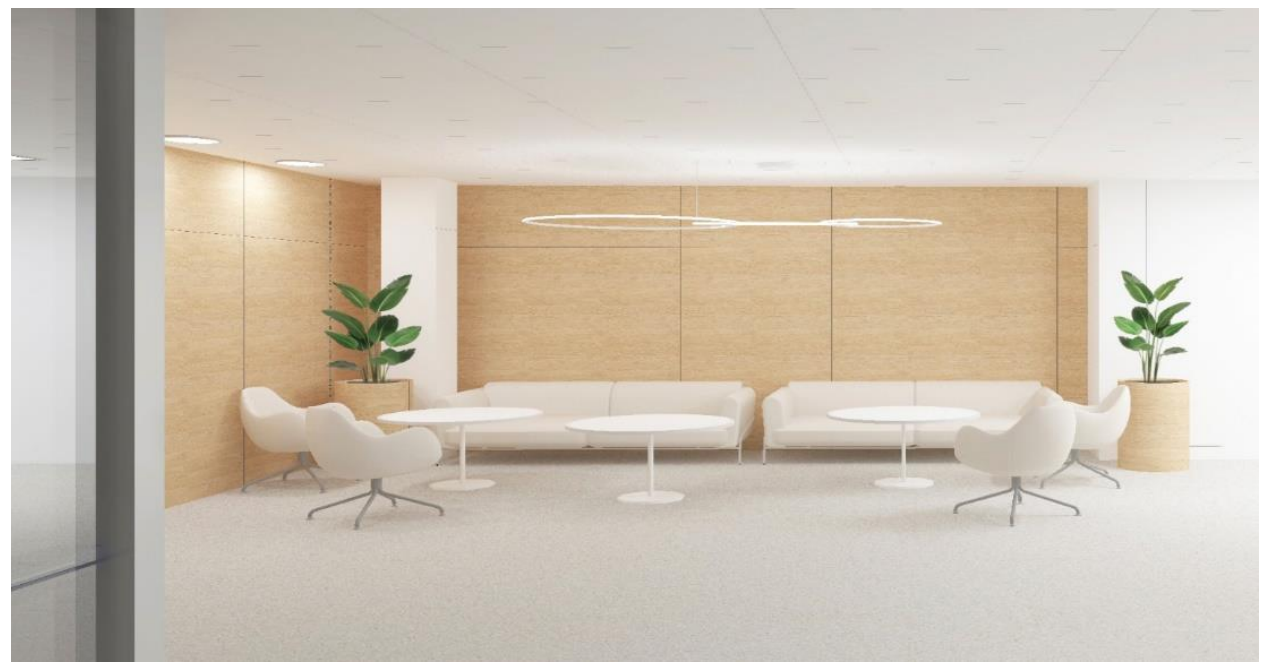

Fig. 2. Lobby. 


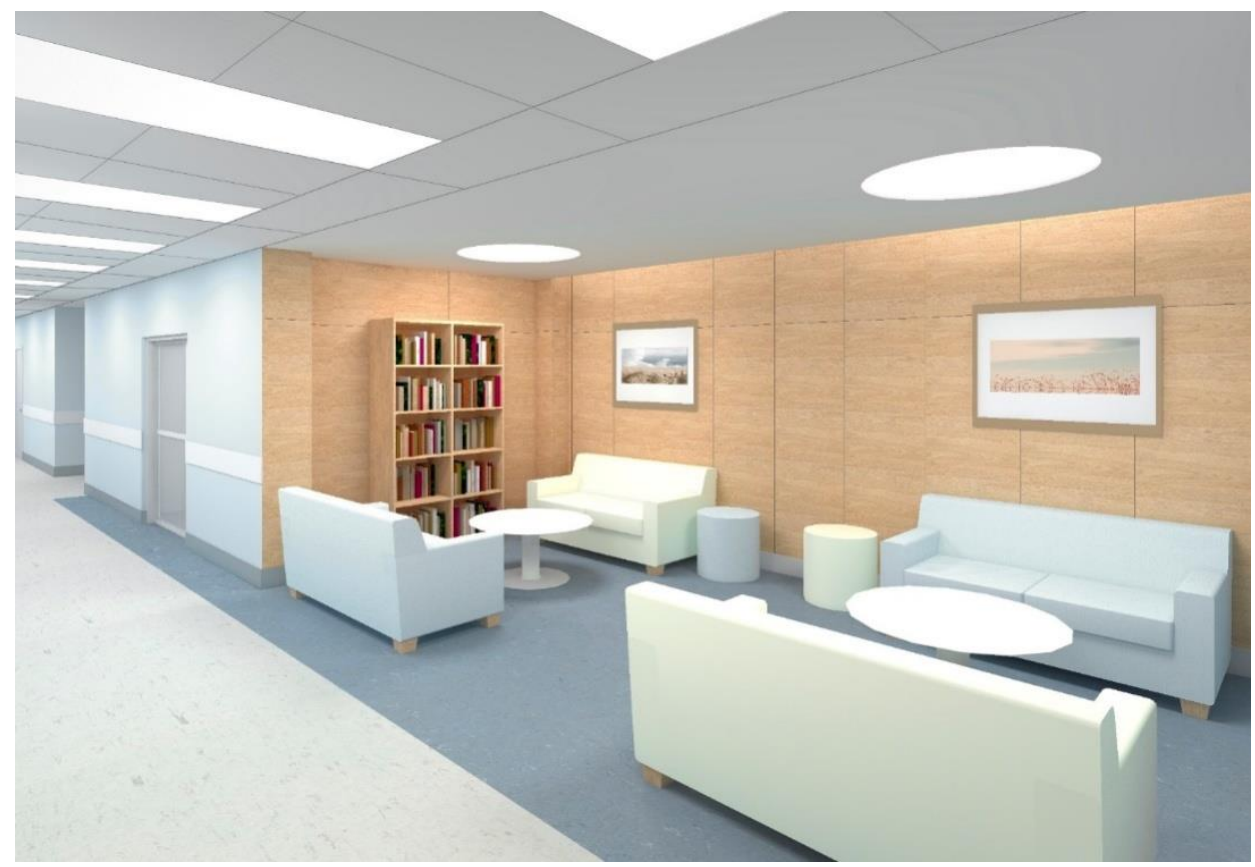

Fig. 3. Waiting area.

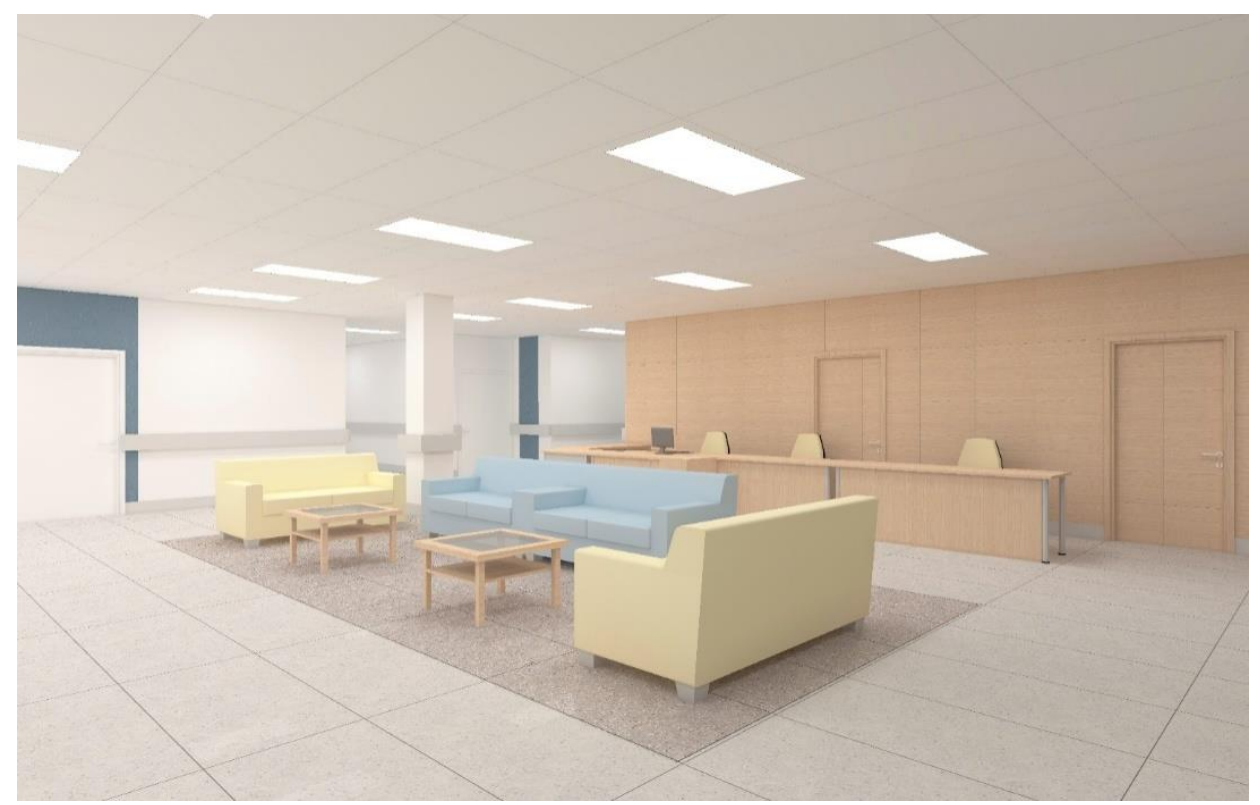

Fig. 4. Day care area. 


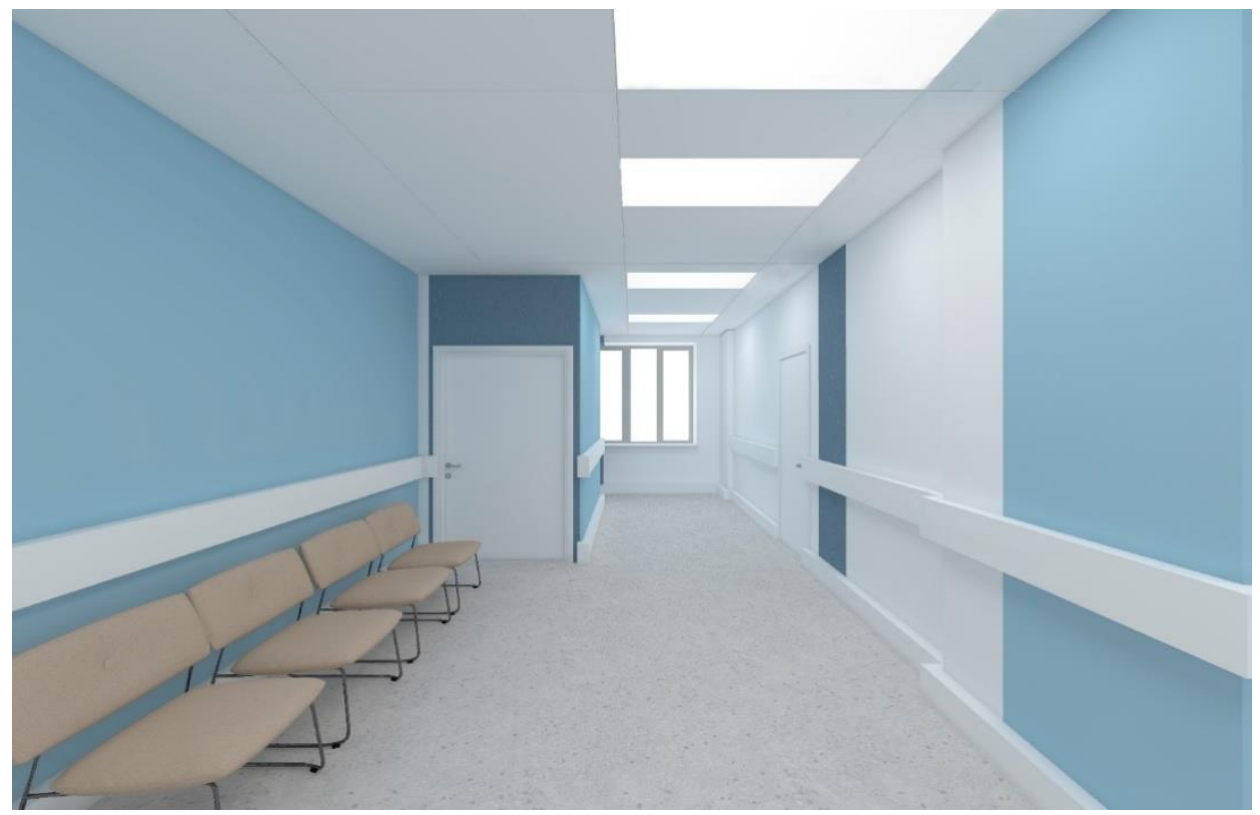

Fig. 5. Corridor.

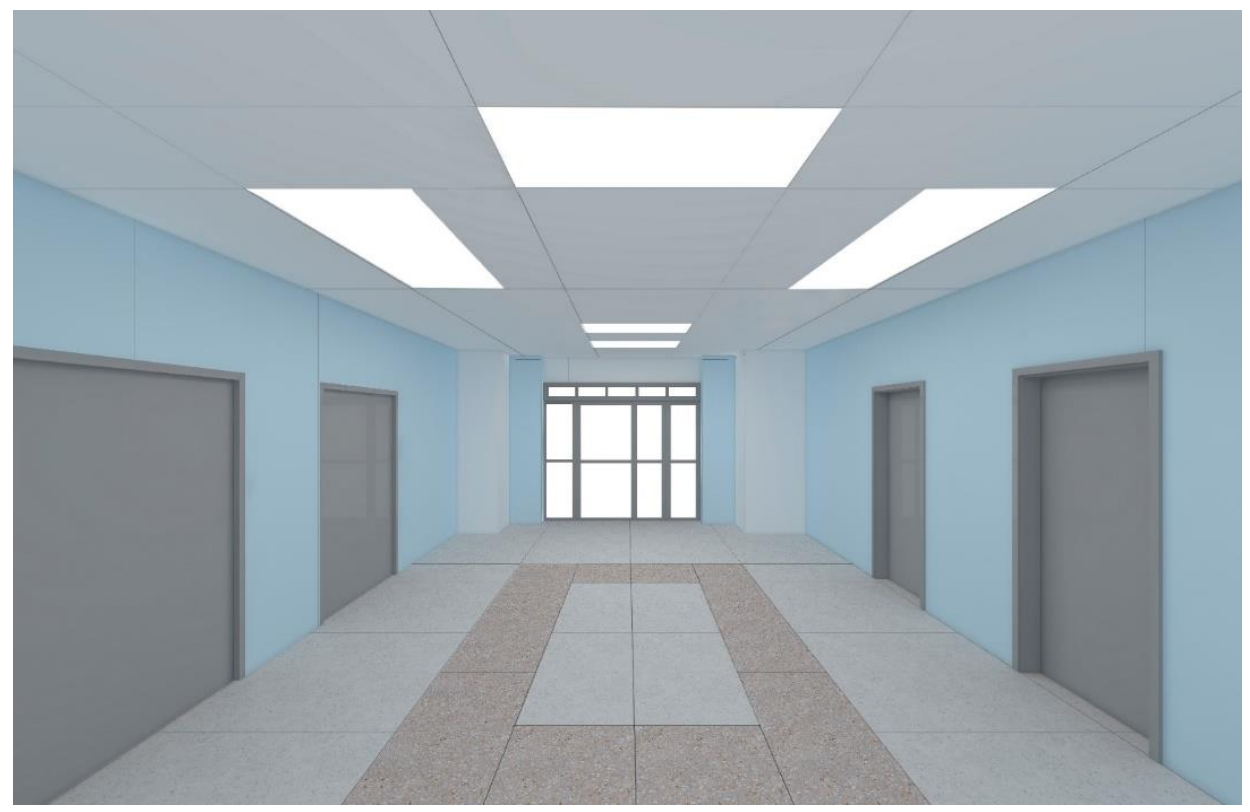

Fig. 6. Lift lobby. 


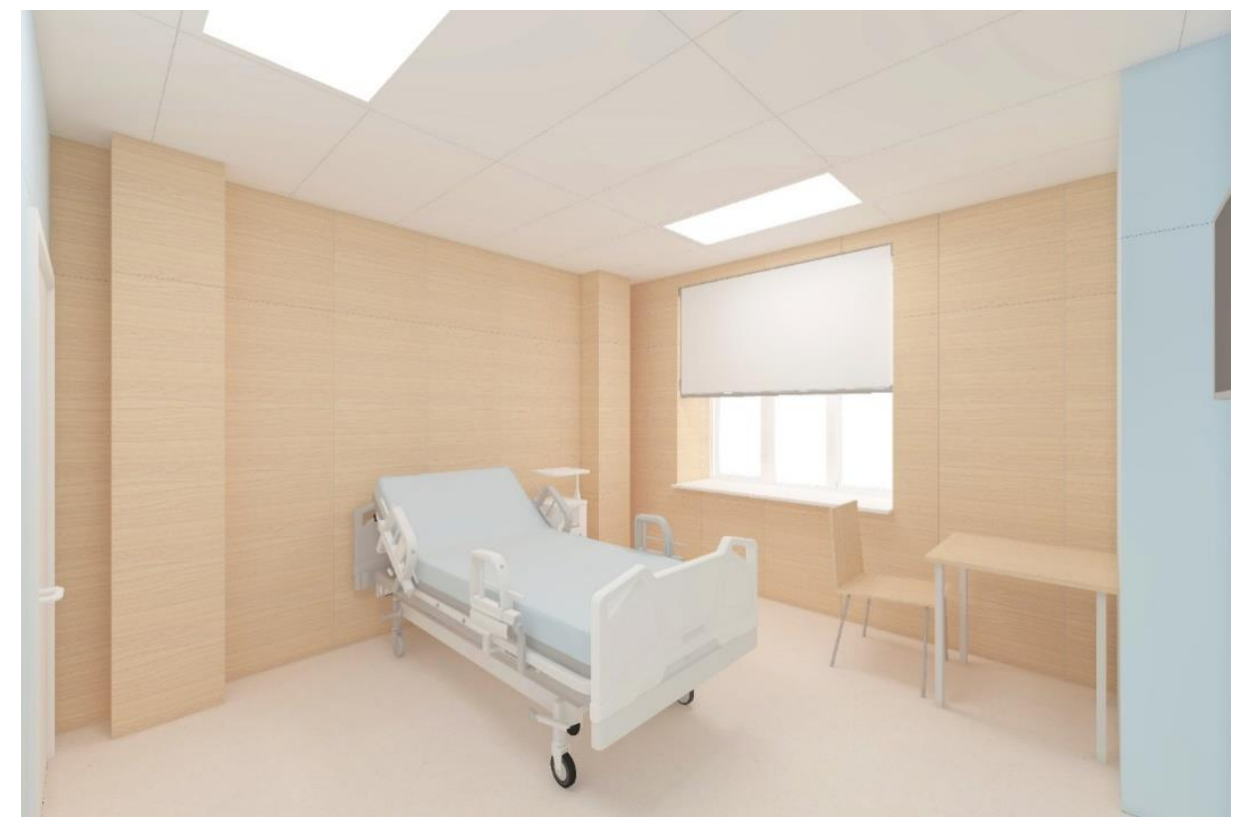

Fig. 7. Ward per 1 bed.

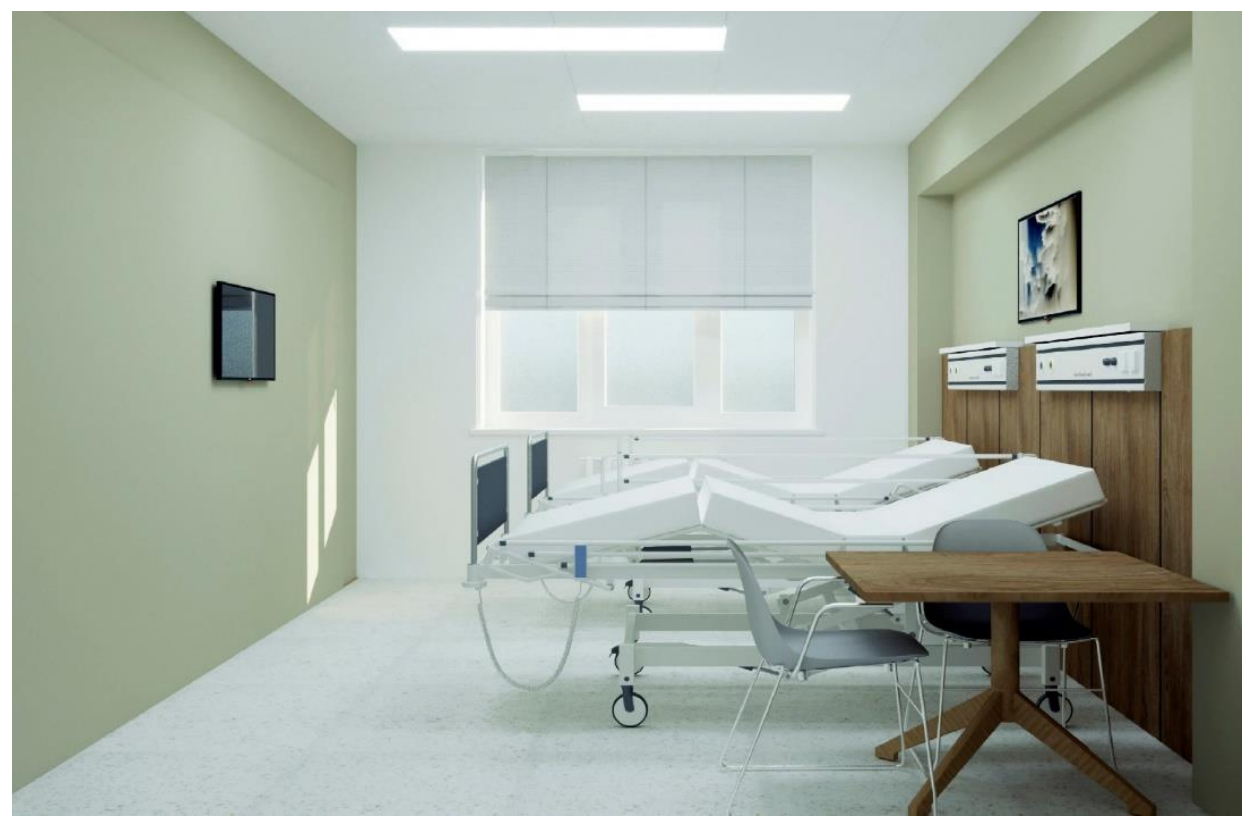

Fig. 8. Ward per 2 beds. 


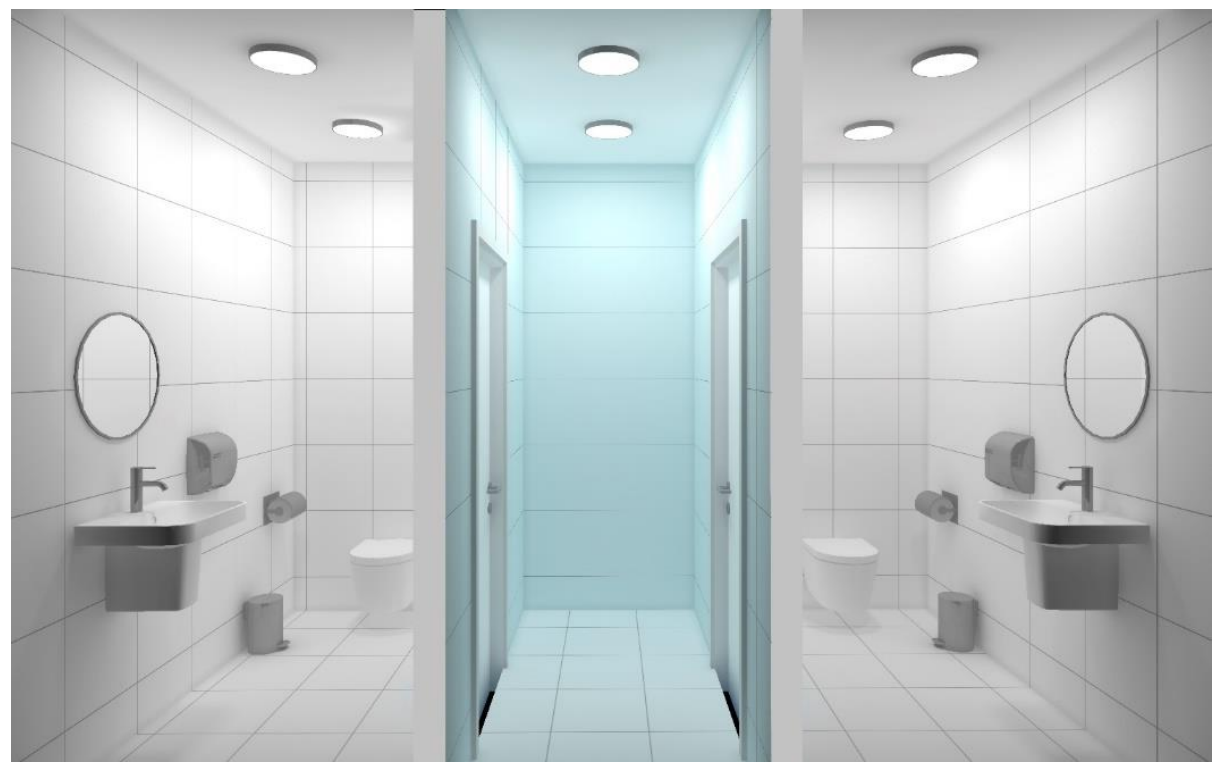

Fig. 9.Sanitary unit.

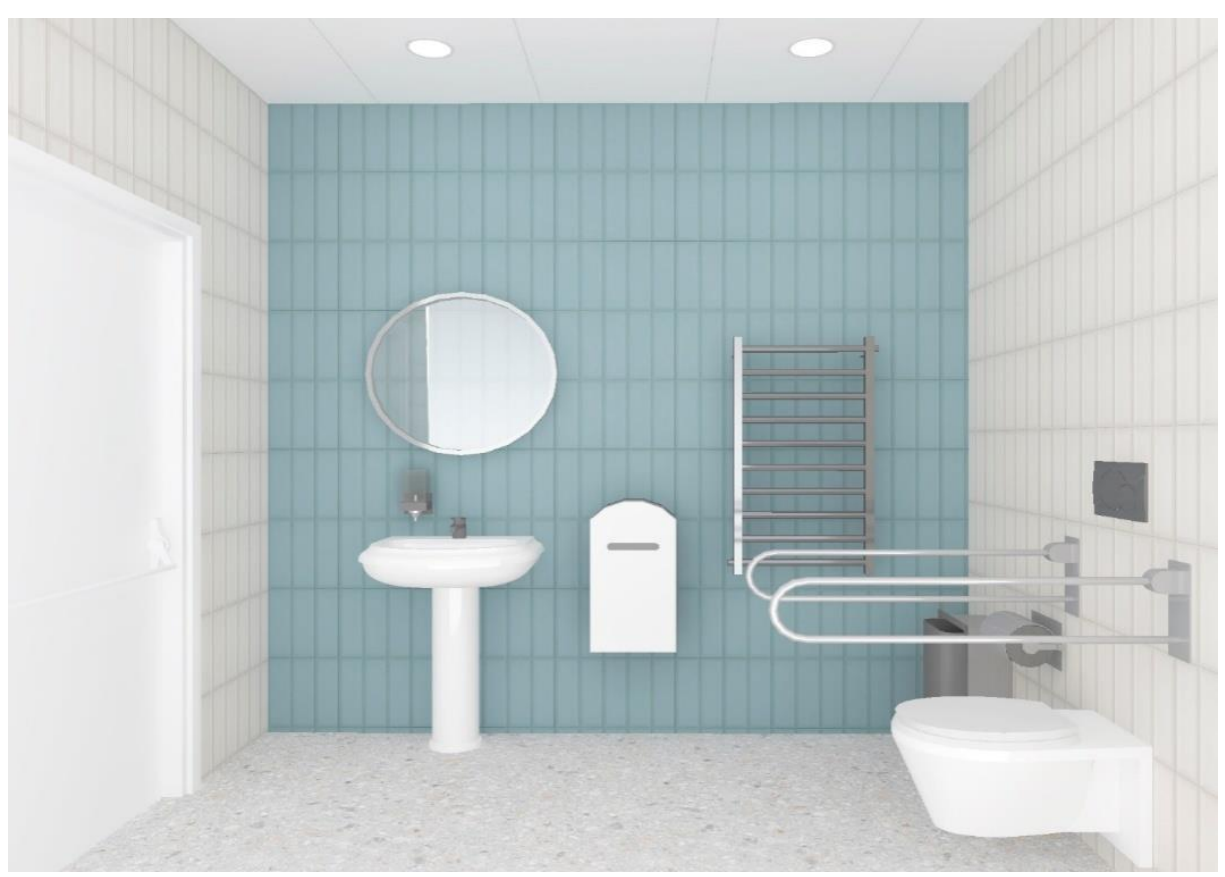

Fig. 10. Sanitary unit for people with low mobility. 


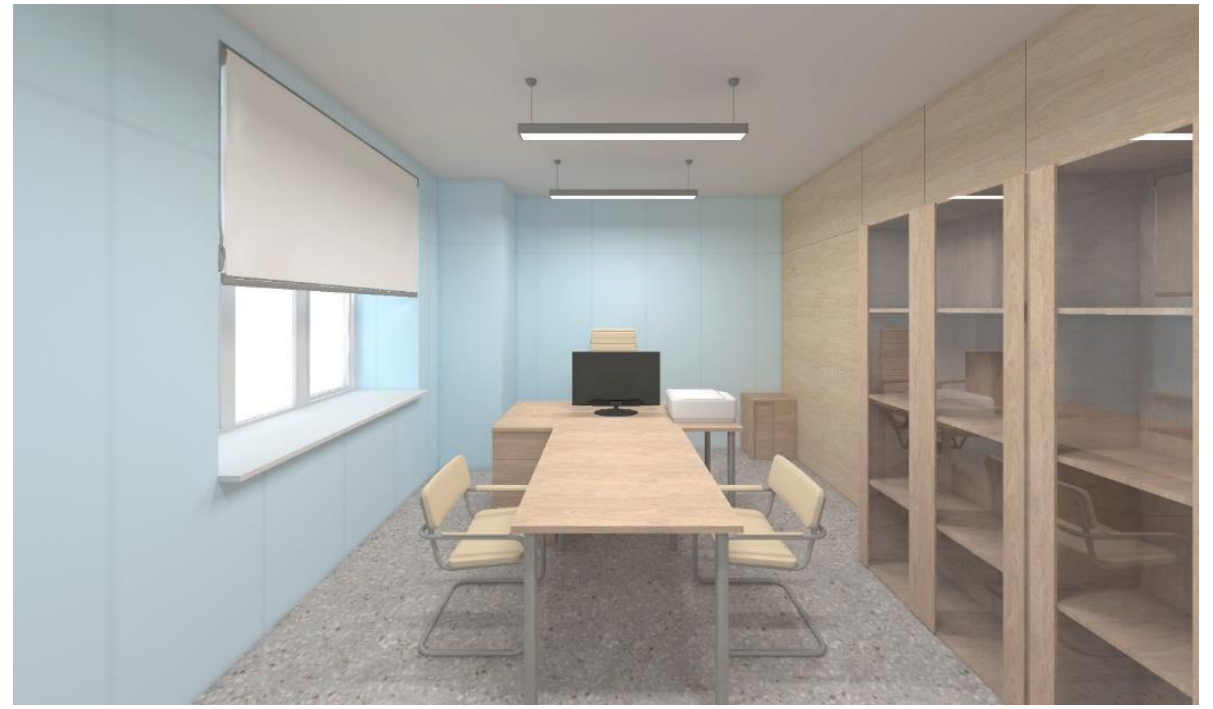

Fig. 11. Inpatient facility head's office.

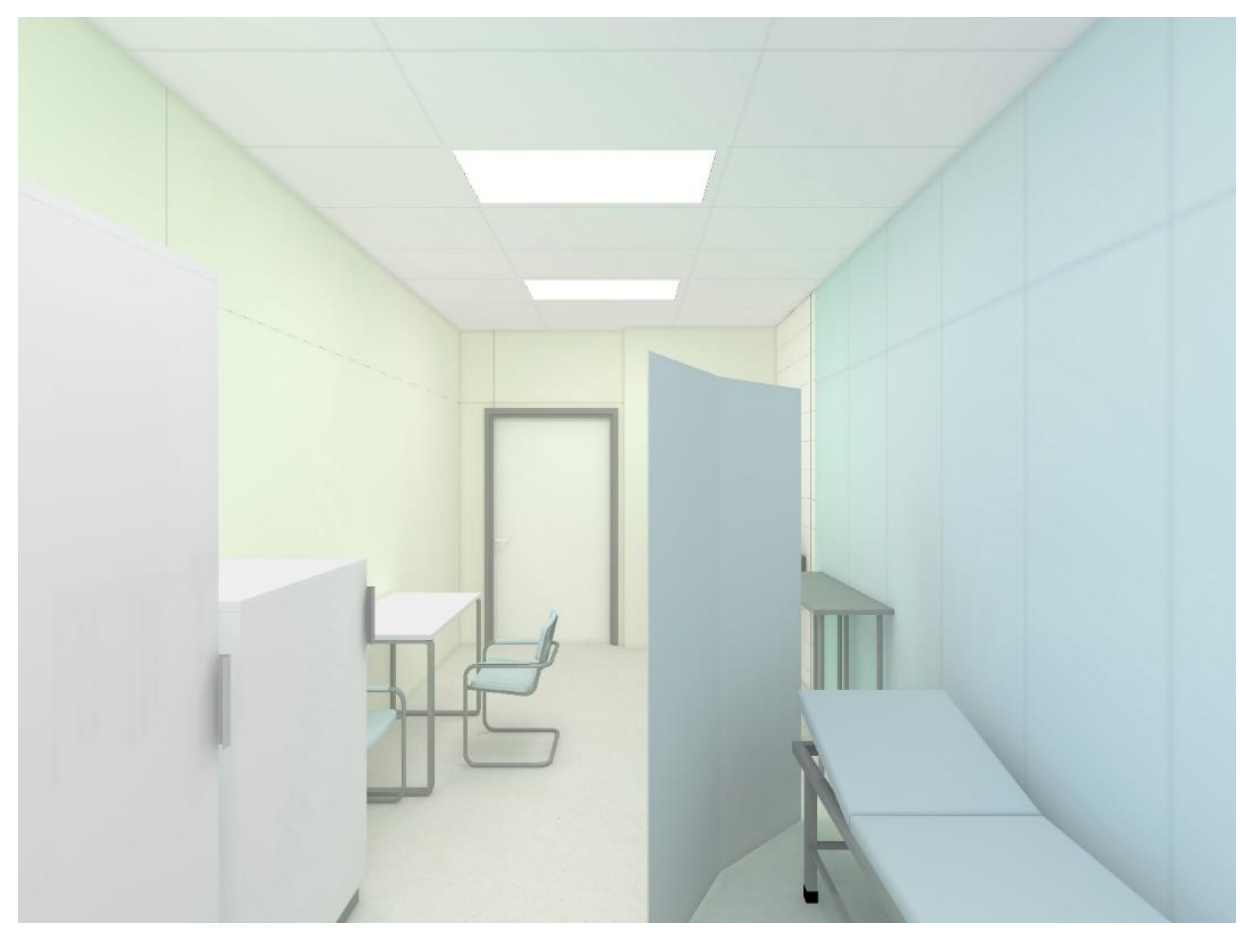

Fig. 12. Examination unit. 


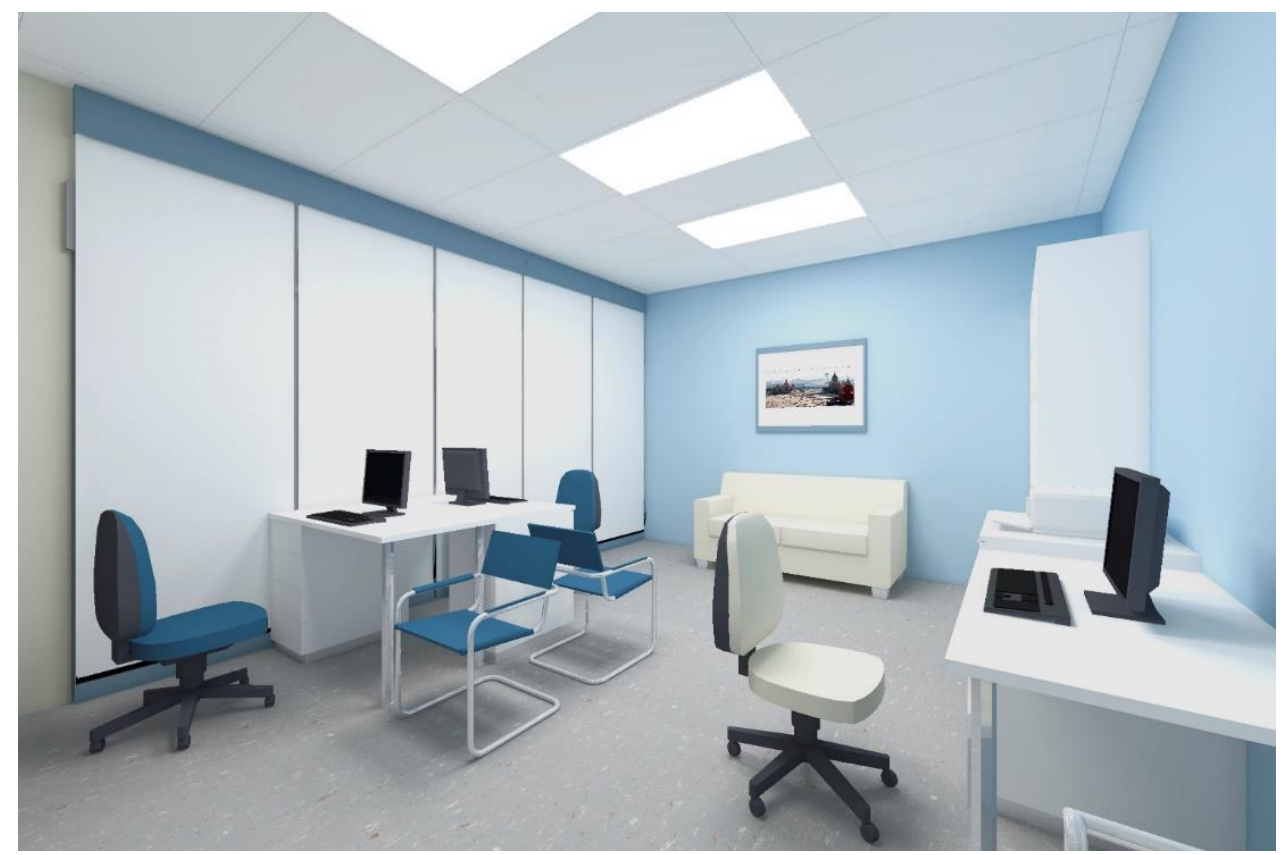

Fig. 13. Doctors' room.

\section{Discussion}

The results of the research allowed us to systematize the requirements for finishing materials of inpatient facility ward units, as well as to determine a pool of finishing materials that fully meet the requirements of regulatory documents, as well as used in construction projects on the capital facilities. The results obtained will simplify the search for appropriate finishing materials used in the interior design of health care institution ward units.

\section{References}

1. Federal Law dated November 21, 2011. No. 323-FZ

2. Order of the Ministry of Health of the Russian Federation dated July 31, 2020. No. $785 n$

3. Russian Federation Standard SP 2.1.3678-20

4. Russian Federation Standard SP 158.13330.2014

5. Russian Federation Standard SP 31-102-99

6. Russian Federation Standard SP 118.13330.2012

7. Russian Federation Standard SNiP 2.08.02-89

8. Preventive Firefighting Regulations 07-91. Fire Safety Rules for Health Care Facilities

9. Y.V. Sorokina, G.I. Rabinovich. Manual to MCCS 4.12-97 Medical and Preventive Treatment Institutions (Moscow Research and Design Institute for Cultural, 2000)

10. Y.V. Sorokina, G.N. Ilnitskaya, E.S. Demina, Manual to MCCS 4.12-97 "Healthcare and Preventive Treatment Facilities"(SUE Moscow Research and Design Institute "Mosproject-4", 2003) 
11. A.I. Arbakov, Manual for Design of Healthcare Facilities to SNiP 2.08.02-89. Section I - General Provisions. Engineering Equipment (1989)

12. A.I. Arbakov, Manual for Design of Healthcare Facilities to SNiP 2.08.02-89). Section II - Inpatient Facilities(1989)

13. GiproNIIzdrav. Recommendations for shaping the interior of hospitals 